\title{
Prevalence of Eating Disorders in Adults with Celiac Disease
}

\author{
V. Passananti, ${ }^{1}$ M. Siniscalchi, ${ }^{2}$ F. Zingone, ${ }^{2}$ C. Bucci, ${ }^{2}$ R. Tortora, ${ }^{1}$ \\ P. Iovino, ${ }^{2}$ and C. Ciacci ${ }^{2}$ \\ ${ }^{1}$ Department of Clinical and Experimental Medicine, University Federico II of Naples, Via S. Pansini, 80131 Naples, Italy \\ ${ }^{2}$ Department of Medicine and Surgery, University of Salerno, Baronissi Campus, Via S. Allende, 84081 Baronissi, Salerno, Italy
}

Correspondence should be addressed to C. Ciacci; cciacci@unisa.it

Received 31 August 2013; Revised 3 November 2013; Accepted 3 November 2013

Academic Editor: R. Eliakim

Copyright (C) 2013 V. Passananti et al. This is an open access article distributed under the Creative Commons Attribution License, which permits unrestricted use, distribution, and reproduction in any medium, provided the original work is properly cited.

\begin{abstract}
Background. Symptoms of celiac disease negatively impact social activities and emotional state. Aim was to investigate the prevalence of altered eating behaviour in celiac patients. Methods. Celiac patients and controls completed a dietary interview and the Binge Eating Staircases, Eating Disorder Inventory (EDI-2), Eating Attitudes Test, Zung Self-Rating Depression Scale, State Trait Anxiety Inventory Forma Y (STAI-Y1 and STAI-Y2), and Symptom Check List (SCL-90). Results. One hundred celiac adults and 100 controls were not statistically different for gender, age, and physical activity. STAI-Y1 and STAI-Y2, Somatization, Interpersonal, Sensitivity, and Anxiety scores of the SLC-90 were higher in CD patients than controls. EDI-2 was different in pulse thinness, social insecurity, perfectionism, inadequacy, ascetisms, and interpersonal diffidence between CD and HC women, whilst only in interceptive awareness between CD and HC men. A higher EAT-26 score was associated with the CD group dependently with gastrointestinal symptoms. The EAT26 demonstrated association between indices of diet-related disorders in both CD and the feminine gender after controlling for anxiety and depression. Conclusion. CD itself and not gastrointestinal related symptoms or psychological factors may contribute pathological eating behavior in celiac adults. Eating disorders appear to be more frequent in young celiac women than in CD men and in HC.
\end{abstract}

\section{Introduction}

Celiac disease (CD) is a chronic inflammatory immunemediated disease. It is a common condition with a prevalence in the western world of about 1:100 [1,2]. Characterized by a clinical heterogeneity, CD presents with a large spectrum of gastrointestinal and extra-intestinal symptoms and it may be diagnosed at any age [3-9]. CD patients also frequently experience somatisation, depression, and anxiety before diagnosis [10-12]. These psychological conditions are in general, associated with changes in appetite, and weight $[13,14]$. A respectable body of data has demonstrated that an association exists between emotional dysfunction and eating disorders [13]. Only few studies, which utilized small sample sizes, though have explored emotional dysfunction and eating disorders in celiac disease $[15,16]$.

The present case-control study was designed to evaluate the prevalence of behavior suggestive of eating disturbances in untreated CD adult patients and to investigate a possible relationship between emotional-psychological factors and the presence of eating disorders.

\section{Methods}

2.1. Study Population. Newly diagnosed CD adults were recruited from outpatient clinics devoted to food intolerances and celiac disease at the Federico II University of Naples and University of Salerno. All patients gave their written informed consent and the study protocol was approved by the Ethic Committee of the University Federico II of Naples (protocol: Diagnosis and Follow-up of Celiac Disease in Adults).

Patients' enrolment lasted from January 2011 to January 2012 and involved all newly diagnosed patients with CD meeting the following inclusion/exclusion criteria.

Inclusion Criteria. Caucasian adults all from the Southern Italian region Campania; age from 18 to 60 years; gastrointestinal symptoms at diagnosis (bloating, abdominal pain, diarrhea); no previous treatment with gluten-free diet; diagnosis of celiac disease according to Corazza-Villanacci histological classification [17] on well-performed biopsies [18], and presence of positive anti-transglutaminase antibodies and antiendomysium antibodies [19]. 
Exclusion Criteria. IgA deficiency, the use of oral contraceptives, oral corticosteroid treatment, hormone replacement therapy, previous diagnosis of eating disorders, presence of other gastrointestinal diseases, pregnancy, diabetes, major psychiatric disorder, physical impairment limiting physical activity, and drug and alcohol abuse. Twenty-six patients were excluded because of presence of one or more exclusion criteria. We enrolled $100 \mathrm{CD}$ patients at diagnosis. One hundred healthy individuals ( $\mathrm{HC}$ ) were enrolled from friends of CD patients and hospital staff and used as controls in comparison with $\mathrm{CD}$ patients. Inclusion criteria for $\mathrm{HC}$ were as follows. Caucasian adults from the Southern Italian region Campania; age from 18 to 60 years; no previous treatment for or diagnosis of celiac disease; negative serum anti-transglutaminase antibodies, and no $\operatorname{IgA}$ deficiency. Exclusion criteria were similar to that of CD patients with the addition of the genetic relationship to a CD patient.

For all participants we collected clinical and anthropomorphic data $\left(\mathrm{BMI}=\right.$ weight $_{\mathrm{kg}} /$ height $\left._{\mathrm{m}}{ }^{2}\right)$. The clinical history included details related to weight reduction/gain, presence of gastrointestinal symptoms (diarrhea, constipation, abdominal pain, bloating, and vomiting), physical activity, and alcohol intake.

2.2. Data Sources. A psychologist (MS) performed a structured psychological assessment and administered the following questionnaires.

(1) EPIC Food Frequency Questionnaire: participants are shown a photographic atlas of 16 food groups. Currently used in epidemiologic studies inSouthern Italy, these pictures depict typical Italian meals in order to help evaluate serving size and eating habits [20]. The food choices of subjects are "measured" with objective criteria helping to recreate food consumption. Although the atlas consists of 64 food/drink tables, patients in this study were only presented with the most frequently used meals in Southern Italy. In addition, we recorded the amount and frequency of consumption of carbonated soft drinks, chocolate and alcohol.

(2) Binge Eating Staircases (BES): BES measures the behavioral aspects of binge eating, as well as the feelings and thoughts associated with such behavior [21]. Originally created to investigate binge-eating behavior in obese patients, the scale has been also validated for nonobese patients and is composed of 16 multiple choice items. Based on the BES total score, individuals can be categorized into three groups according to established cut scores of binge eating severity [22]. A frequent convention is to use the BES as a screening measure to classify all participants with scores greater than or equal to 17 as "binge eaters" [23].

(3) Eating Attitudes Test (EAT-26): EAT-26 is a questionnaire with 26 items [24]. It investigates diet-related disorders, bulimia, and anxiety related to food. More specifically, EAT-26 highlights the perception of one's own weightand physical appearance. The EAT-26 test is a standardized and validated screening tool that can be considered a valuable aid in the diagnosis of eating disorders cross-culturally. Each question is scored on a scale of 0 to 3 (based on the replies: always, usually, often, sometimes, rarely, and never). A total score is obtained by adding the scores of each item. Possible scores on the EAT-26 range from 0 to 78. A score of 20 or above indicates that a person may have an eating disorder and is recommended for further evaluation by a mental health professional.

(4) Eating Disorder Inventory (EDI-2) [25]: EDI-2 is a selfassessment instrument of the symptoms associated with anorexia nervosa and bulimia nervosa. In particular, it can be used to identify patients with "masked" eating disorders. The test can also detect and accurately measure psychological aspects or symptoms relevant to the treatment of eating disorders. The EDI2 consists of 91 items and 11 scales and measures the following constructs: pulse thinness, bulimia, dissatisfaction for own body, interpersonal diffidence, perfectionism, inadequacy, interceptive awareness, fear of maturity, asceticism, impulsivity, and social insecurity. Each item is scored from 1 to 6: "always," "usually," "often" or "sometimes," "rarely," and "never." We used the Italian version of EDI-2.

(5) Italian Version of the Original Zung Self-Rating Depression Scale (M-SDS) [26, 27]: M-SDS investigates the presence of depressive symptoms. The MSDS contains 20 items. In the M-SDS, a score of 44 is the cut-off value for pathological depression.

(6) STAI-Y1 and STAI-Y2: are these scales which aim to measure the presence and grade of anxiety [28]. The questionnaires are each 20 items, with responses related to terms of intensity (from "almost never" to "almost always"). The items are grouped into two axes, which permit a distinction between existing anxiety (STAI-Y1) and predisposition to an anxious reaction as a personality characteristic (STAI-Y2). A score $\geq 40$ is the cut-off value for both scales.

(7) Symptom Check List (SCL-90): SCL-90 is a self-administered questionnaire, consisting of 90 items. SCL90 assesses a wide range of psychological and psychopathological symptoms by measuring internalizing (depression, somatization, and anxiety) and externalizing symptoms (aggression, hostility, and impulsivity). The items are grouped into 10 clusters (Somatization (SOM), Obsessive-compulsive (OC), Interpersonal Sensitivity (INT), Depression, (DEP) Anxiety (ANX), anger-hostility (HOS), phobic anxiety (PHOB), psychoticism (PSY), paranoid ideation (PAR), and sleep disturbances (SLEEP)). For each item, the patient responds using a scale of severity from 0 to 4 , in reference to the last week [29].

2.3. Statistical Analysis. Percent frequencies and means with respective standard deviations were calculated for sample descriptive statistics 
TABLE 1: Basic characteristics of the study population.

\begin{tabular}{lccc}
\hline & CD & HC & $P$ \\
\hline Number of women & 72 & 68 & 0.6 \\
Age (means \pm SD) & $29.2 \pm 8.7$ & $30.1 \pm 8.2$ & 0.5 \\
BMI $\left(\mathrm{Kg} / \mathrm{m}^{2}\right)$ & $\mathbf{2 2 . 0} \pm \mathbf{3 . 5}$ & $\mathbf{2 3 . 3 0 \pm \mathbf { 3 . 0 }}$ & $\mathbf{0 . 0 0 5}$ \\
$\begin{array}{l}\text { \% Reporting alcohol intake } \\
\text { \% Reporting moderate to high } \\
\text { physical activity }\end{array}$ & 15 & 16 & 1.0 \\
$\begin{array}{l}\text { \% Reporting gastrointestinal } \\
\text { symptoms }\end{array}$ & $\mathbf{7 8}$ & 36 & 0.7 \\
\hline
\end{tabular}

Data showed in bold are significantly different $(P<0.05)$ between groups. $\mathrm{CD}$ : celiac disease group; HC: healthy controls group.

Unpaired Student's $t$-test was used to compare continuous data and $\chi^{2}$ for categorical data. Univariate and multivariate regression analyses were performed as appropriate. The statistical program used was the Statistical Package for the Social Sciences (SPSS) for Windows, version 12.0. Statistical significance was accepted as $P<0.05$.

\section{Results}

3.1. Study Population. All eligible CD patients $(n=100)$ and HC $(n=100)$ accepted to undergo psychological assessment and administration of questionnaires. Table 1 depicts basic characteristics of the study population. CD patients were not significantly different from HC in regard to gender and age. Those in the CD group had a significantly lower BMI as compared to healthy controls. The percentage of individuals reporting moderate to high level of physical activity and percentage of subjects reporting some alcohol intake were not significantly different between the two groups $(P=0.7$ and 1.0, resp.). As expected, CD patients had a significantly higher prevalence of gastrointestinal symptoms compared to that of HC's (Table 1).

\subsection{Eating Behaviors}

3.2.1. EPIC Food Frequency Questionnaire. The photographic atlas for assessing quality and quantity of food intake showed that fish and meat meal portions were similar between both groups. The overall carbohydrate intake was greater in CD patients compared to $\mathrm{HC}$ and this difference was statistically significant $(P<0.05)$. Specifically, the consumption of pasta and bread was approximately $30 \%$ higher in CD than in HC. Although the consumption of fruits and vegetables was slightly lower in CD than in HC, this difference was not statistically significant.

3.2.2. Binge Eating Staircases. The percentage of pathological BES scores was similar in CD and HC groups (6\% versus $0 \%$; $P=0.09)$. In men BES pathological scores of CD compared with $\mathrm{HC}$ were statistical significant different (6\% versus $0 \%$ $P=0.04)$. Zero women in the $\mathrm{CD}$ or $\mathrm{HC}$ groups had pathological scores for BES.

3.2.3. Eating Attitudes Test (EAT-26). A significantly higher EAT-26 score was found in the CD group compared to $\mathrm{HC}$
TABLE 2: Profile of celiac disease (CD) and healthy controls (HC) groups according to EDI-2 scores in women in Table 2(a) and men in Table 2(b).

(a)

\begin{tabular}{lccc}
\hline EDI-2 women & $\mathrm{CD}$ & $\mathrm{HC}$ & $P$ \\
\hline Pulse thinness & $\mathbf{6 . 1} \pm \mathbf{4 . 2}$ & $\mathbf{2 . 6} \pm \mathbf{2 . 6}$ & $\mathbf{0 . 0 0 1}$ \\
Bulimia & $1.2 \pm 2.0$ & $2.0 \pm 2.6$ & $\mathrm{Ns}$ \\
Dissatisfaction for own body & $7.2 \pm 5.9$ & $6.1 \pm 5.3$ & $\mathrm{Ns}$ \\
Interpersonal diffidence & $\mathbf{4 . 2} \pm \mathbf{3 . 4}$ & $\mathbf{2 . 2} \pm \mathbf{1 . 8}$ & $\mathbf{0 . 0 1}$ \\
Perfectionism & $\mathbf{5 . 0} \pm \mathbf{2 . 9}$ & $\mathbf{3 . 1} \pm \mathbf{3 . 2}$ & $\mathbf{0 . 0 3}$ \\
Inadequacy & $\mathbf{5 . 2} \pm \mathbf{4 . 4}$ & $\mathbf{2 . 2} \pm \mathbf{2 . 2}$ & $\mathbf{0 . 0 3}$ \\
Interceptive awareness & $3.6 \pm 3.2$ & $2.2 \pm 3.5$ & $\mathbf{N s}$ \\
Fear of maturity & $3.4 \pm 3.5$ & $3.7 \pm 2.9$ & $\mathbf{N s}$ \\
Asceticism & $\mathbf{5 . 9} \pm \mathbf{4 . 5}$ & $\mathbf{2 . 2} \pm \mathbf{2 . 0}$ & $\mathbf{0 . 0 0 1}$ \\
Impulsivity & $3.4 \pm 4.0$ & $2.3 \pm 3.4$ & $\mathbf{N s}$ \\
Social insecurity & $\mathbf{6 . 7} \pm \mathbf{5 . 3}$ & $\mathbf{3 . 0} \pm \mathbf{2 . 6}$ & $\mathbf{0 . 0 0 3}$ \\
\hline
\end{tabular}

Data are expressed as means (M) and standard deviation (SD).

Data showed in bold are significantly different $(P<0.05)$ between groups. Ns: not statistically significant; CD: celiac disease group; HC: Healthy controls group.

(b)

\begin{tabular}{lccc}
\hline EDI-2 men & CD & HC & $P$ \\
\hline Pulse thinness & $1.4 \pm 1.3$ & $3.3 \pm 2.3$ & Ns \\
Bulimia & $2.2 \pm 3.9$ & $1.7 \pm 1.2$ & Ns \\
Dissatisfaction for own body & $4.4 \pm 2.8$ & $4.4 \pm 2.0$ & Ns \\
Interpersonal diffidence & $2.8 \pm 1.5$ & $1.4 \pm 1.7$ & Ns \\
Perfectionism & $3.4 \pm 2.2$ & $1.6 \pm 1.3$ & Ns \\
Inadequacy & $4.0 \pm 4.3$ & $2.6 \pm 2.3$ & Ns \\
Interceptive awareness & $\mathbf{4 . 0} \pm \mathbf{2 . 9}$ & $\mathbf{1 . 1} \pm \mathbf{1 . 5}$ & $\mathbf{0 . 0 2}$ \\
Fear of maturity & $4.2 \pm 6.3$ & $2.6 \pm 1.7$ & Ns \\
Ascetisms & $3.4 \pm 3.5$ & $2.3 \pm 2.0$ & Ns \\
Impulsivity & $2.2 \pm 2.3$ & $2.7 \pm 2.1$ & Ns \\
Social insecurity & $4.4 \pm 4.8$ & $3.1 \pm 3.2$ & Ns \\
\hline
\end{tabular}

Data are expressed as means (M) and standard deviation (SD). Data showed in bold are significantly different $(P<0.05)$ between groups.

Ns: not statistically different.

but was not independently associated with gastrointestinal symptoms (regression coefficient, $B=3.7$, SE 1.7, $P=0.03$ ). The percentage of pathological EAT-26 scores is significantly different between $\mathrm{CD}$ and $\mathrm{HC}$ groups (16\% versus $4 \%$; $P=$ $0.01)$.

3.2.4. Eating Disorder Inventory (EDI-2). Table 2 profiles CD and $\mathrm{HC}$ groups according to EDI-2 scores in women and men. The EDI-2 questionnaire was originally validated only for young adults (16 to 26 years). Therefore, in order to avoid any age-related bias, analysis was repeated for CD and HC subgroups (n.76) with ages 18 to 26 years. Results were comparable to what was found in the entire cohort (data not shown). Compared to HC, CD women had significant differences in several items such as pulse thinness, social insecurity, perfectionism, inadequacy, ascetisms, and interpersonal diffidence compared to $\mathrm{HC}$ women, whilst CD men 


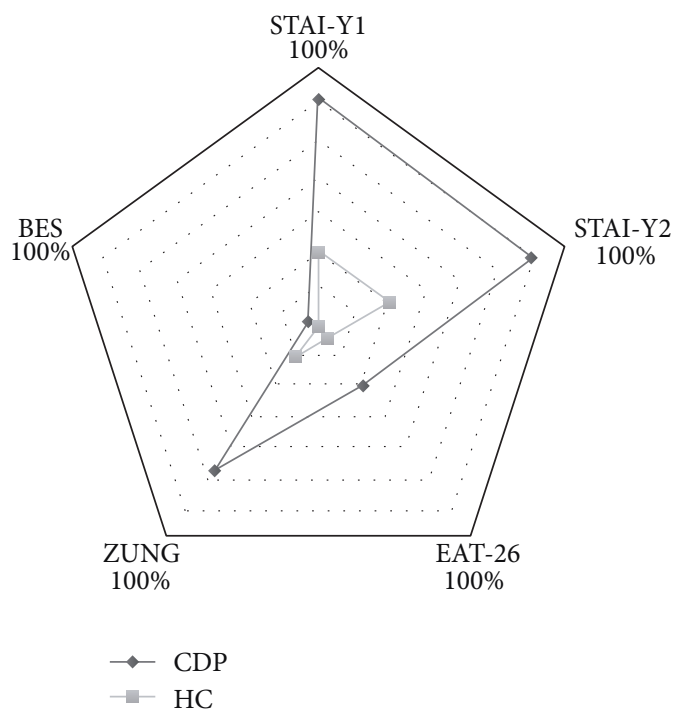

FIGURE 1: Graphic visualization of percentages of pathological scores of BES, M-SDS, EAT-26, STAY1, and STAY2 scales in both groups.

were different from $\mathrm{HC}$ men only for interceptive awareness. Eleven CD patients and $1 \mathrm{HC}$ scored more than 20 items of the EDI-2 questionnaire. They were further interviewed (MS) and a clear eating disorder diagnosis was confirmed only in the $11 \mathrm{CD}$ patients.

3.2.5. M-SDS, STAY-1 and STAY-2, and SLC-90. Figure 1 is a representation diagram that includes the results (expressed as percentage of pathological scores) of M-SDS, EAT-26, STAY1 and STAY2, and BES. Thirty-nine percent of CD patients and $6 \%$ of HC reported a pathological M-SDS $\left(\chi^{2} 29.362, P<\right.$ $0.001)$. STAY1 and STAY2 were also statistically significantly different between CD and HC groups (59\% versus $18 \%, \chi^{2}$ $0.787, P<0.001$ and $61.4 \%$ versus $18.4 \%, \chi^{2} 33.908, P<$ 0.001 , resp.). Due to the higher prevalence of women in both groups, a gender analysis was performed for M-SDS and STAY; a significantly higher prevalence of the prevalence of pathological scores for M-SDS, STAY1 and STAY2 was significantly higher $\mathrm{CD}$ women than in $\mathrm{HC}$ women. Men in the $\mathrm{CD}$ group also had a significantly higher pathological score frequency for M-SDS and STAY1 than in the HC (Table 3).

Both global and pathological scores from SLC-90 were significantly different between $\mathrm{CD}$ and $\mathrm{HC}$ (pathological scores: $42 \%$ versus $6 \%, P<0.001$ ). Comparison of single items revealed statistically different pathological values between $\mathrm{CD}$ patients and $\mathrm{HC}$ for somatization, obsessivecompulsive, interpersonal sensitivity, depression, anxiety, and sleep disorders (Figure 2). Furthermore, when stratifying by gender in the CD group; somatisation, obsessive-compulsive, interpersonal sensitivity, depression, anxiety, and sleep disorders scales were more frequently pathological in women than men (Figure 3).

A multivariate analysis was performed in order to determine the independent effects of $\mathrm{CD}$ and gender on eating
TABLE 3: The percentages of pathological M-SDS, STAI-Y1, and STAI-Y2 scale scores in celiac disease patients (CDP) and healthy controls (HC) according to gender.

\begin{tabular}{lccc}
\hline & CD & HC & $P$ \\
\hline M-SDS & & & \\
Men & $\mathbf{3 0 \%}$ & $\mathbf{0 \%}$ & $\mathbf{0 . 0 2 0}$ \\
Women & $\mathbf{5 0 \%}$ & $\mathbf{1 4 . 7 \%}$ & $\mathbf{0 . 0 0 2}$ \\
STAI-Y1 & & & \\
Men & $\mathbf{4 0 \%}$ & $\mathbf{6 . 3 \%}$ & $\mathbf{0 . 0 3 4}$ \\
Women & $\mathbf{6 4 . 7 \%}$ & $\mathbf{2 3 . 5 \%}$ & $\mathbf{0 . 0 0 1}$ \\
STAI-Y2 & & & \\
Men & $40 \%$ & $12.5 \%$ & Ns \\
Women & $\mathbf{6 7 . 6 \%}$ & $\mathbf{2 1 . 2 \%}$ & $\mathbf{0 . 0 0 0 1}$ \\
\hline
\end{tabular}

Data are expressed as percentages and $P$ value (Student's $t$-test for unpaired data).

Data showed in bold are significantly different $(P<0.05)$ between groups. Ns: not statistically significant; CD: celiac disease group; HC: healthy controls group.

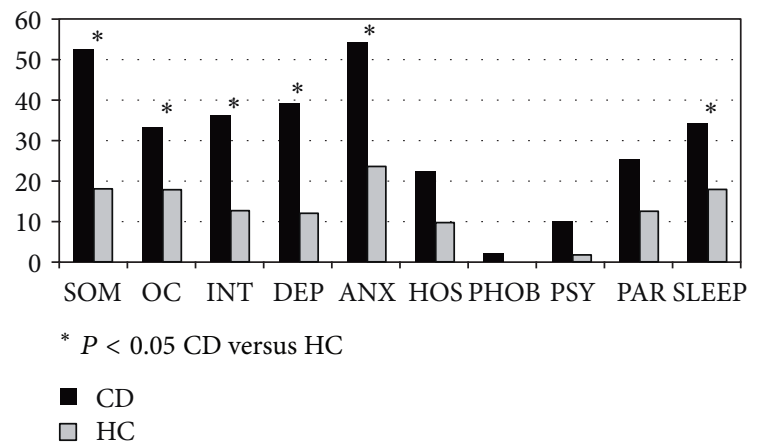

Figure 2: Percentages of pathological SLC-90 subscale scores in celiac disease (CD) patients (black) and healthy controls (HC, grey). Data are expressed as percentages and $P$ value (Student's $t$-test for unpaired data).

disorder behaviour. EAT-26 scores, as a surrogate of eating disorder behaviour, were modelled against gender and CD, using M-SDS, STAY1 and STAY2 as covariates. A significant association between EAT-26 score and both CD presence $(P=0.02)$ and feminine gender $(P=0.003)$ without any significant influence of anxiety and depression was found when incorporating M-SDS, STAY1 and STAY2 into the model. In particular, EAT-26 scores were 3.9 units lower in $\mathrm{HC}$ than $\mathrm{CD}$ after adjusting for depression and anxiety (B -3.9 95\% [CI -7.2/-0.5], $P=0.03)$; In the adjusted model, EAT-26 scores were 5 units lower in males than in females $(B-5.0595 \%$ [CI $-9.5 /-0.5], P=0.02)$. Overall, our results indicate that eating disorder behavior, through EAT-26 scores, was significantly higher in CD patients and in women, whilst there seems to be no significant correlation with the presence of gastrointestinal symptoms.

\section{Discussion}

This study systematically investigates the prevalence and characteristics of eating behaviors in untreated celiac patients and healthy controls through the use of several validated 


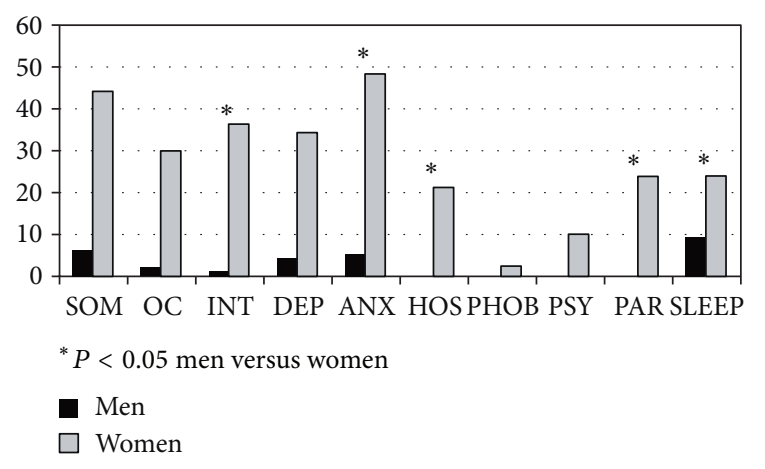

Figure 3: Percentages of pathological SLC-90 subscale scores in celiac disease women and men. $*$ indicate subscales with statistically significant differences $(P<0.05)$ between the two sexes (Student's $t$-test for unpaired data).

questionnaires. Our findings indicate that the frequency of altered eating behavior is increased in untreated $C D$ when compared with HC. Our data also show that bulimic behavior was found only in CD men. Another corollary, yet relevant datum, is that untreated CD patients, both men and women, report a higher daily carbohydrate intake (that in Southern Italy is mainly due to pasta and bread consumption) in comparison to $\mathrm{HC}$ and this finding may support the hypothesis of high gluten intake before the onset of celiac disease in genetically predisposed individuals.

To our knowledge, this study is the first that shows the presence of altered eating behaviors in untreated CD.

The EAT-26 is probably the most used questionnaire to measure symptoms and concerns due to eating disorders. EAT-26 is considered only a first step in the evaluation of a patient followed in case of pathological scores by an interview by qualified professional to assess if the individual meets the criteria for an eating behavior diagnosis. There were no significant differences in the frequency of pathological EAT26 between $\mathrm{CD}$ and $\mathrm{HC}$. However, most of CD patients scored more than 10 items in the EAT-26 scale, not enough to a precise diagnosis but enough to underline a trend toward a strong concern about body and food intake. Regression analyses demonstrated that high scores of EAT-26 in CD do not correlate with presence of gastrointestinal symptoms or with high scores of anxiety and depression scales.

The EDI-2 scale, that is, a self-assessment of symptoms associated with anorexia nervosa and bulimia nervosa showed that CD men were significantly different from HC in the interceptive awareness, that is, the measure of the ability of an individual to discriminate between sensations and feelings and between hunger and satiety. CD women showed higher scores than HC women for pulse thinness, ascetism, perfectionism, and inadequacy. These items suggest the profile of body dissatisfaction, insecurity in one's physical appearance, and strong feelings of inadequacy linked to tendency to the avoidance of sexual relationships, and in general feelings of social insecurity.

The increased frequency of eating behavior in untreated $\mathrm{CD}$ might be a consequence of a number of concurrent factors.
Firstly, it might be a feature of the already known altered psychological status of CD $[4,30]$. However, in our series the multivariate analysis between EAT-26 and STAY-1 and STAY-2 and M-SDS scales did not disclose any relationship between eating disorders and anxiety and depression indices.

Secondly, an increased frequency of eating disorder could be food-related as carbohydrate intake has been shown to be higher in CD than in HC. In fact, high carbohydrate intakes are related to the presence of gastrointestinal symptoms [30]. Therefore, it is possible that gastrointestinal symptom onset subsequent to food intake (particularly those containing gluten and/or high content of unadsorbable carbohydrates, i.e., FODMAPs) might be a relevant conditioner in altered eating habits in $\mathrm{CD}$ [31].

Thirdly, CD patients commonly suffer concomitant functional bowel disorders, as shown by the persistence of gastrointestinal symptoms after initiation of a consolidated strict GFD [32]. However, our data indicate that eating disorders do not correlate with the presence of gastrointestinal symptoms in $\mathrm{CD}$ group making this explanation unlikely.

Lastly, it is hypothesized that hormones or other mediators in the blood stream might play a role in the genesis of altered eating behavior in $\mathrm{CD}$ [33].

Previous studies related to $\mathrm{CD}$ and eating disorders have been limited to only a small number of patients. Currently, through the utilization of a large case control study, we demonstrated that the emotional relationship of untreated celiac patients with food is altered and significantly different from that of a control population. In this study, selection bias was also limited as patients were recruited from a dedicated clinic at the moment of diagnosis, before receiving the prescription to obtain gluten-free food under the coverage of the National Health System.

The main limitation of this study relates to the lack of information on the effect of gluten-free diet on eating disorders (study is in progress). Additionally, collection of information on dietary habits may have been subject to possible recall and observation bias.

In conclusion, the findings of this study further delineate the importance of caregiver vigilance regarding the recognition of eating disorders in celiac patients.

\section{Authors' Contribution}

Each author certifies that he or she has participated sufficiently in the intellectual content, the analysis of data, if applicable, and the writing of the work to take public responsibility for it. Each has reviewed the final version of the work, believes it represents valid work, and approves it for publication. Moreover, should the editors of the Publication request the data upon which the work is based, they shall produce it.

\section{Disclaimer}

Each author warrants that this work contains no libelous or unlawful statements and does not infringe on the rights of others. If excerpts (e.g., text, figures, tables, illustrations, or audio/video files from copyrighted works are included), 
a written release will be secured by the authors prior to submission, and credit to the original publication will be properly acknowledged. Each author warrants that he or she has obtained, prior to submission, written releases from patients whose names or likenesses are submitted as part of the work.

\section{Disclosure}

Each author certifies that he or she has no commercial associations (e.g., consultancies, stock ownership, equity interest, patent/licensing arrangements, etc.) that might pose a conflict of interest in connection with the work, except as disclosed on a separate attachment. Each author warrants that his or her submission to the work is original and that he or she has full power to enter into this agreement. Neither this work nor a similar work has been published nor shall be submitted for publication elsewhere while under consideration by this Publication. Except for copyright, other proprietary rights related to the work shall be retained by the authors, to reproduce any text, figures, tables, or illustrations from this work in future works of their own.

\section{Ethical Approval}

Each author certifies that his or her institution has approved the protocol for any investigation involving humans and that all experimentation was conducted in conformity with ethical and humane principles of research.

\section{Acknowledgments}

This study has been supported by the Regione Campania educative project Le Patologie della nutrizione e del metabolismo nell'adulto in Campania. The authors are grateful to Daniel DiGiacomo, Tulane University School of Medicine, for his review of the paper.

\section{References}

[1] A. Fasano, I. Berti, T. Gerarduzzi et al., "Prevalence of celiac disease in at-risk and not-at-risk groups in the United States: a large multicenter study," Archives of Internal Medicine, vol. 163, no. 3, pp. 286-292, 2003.

[2] K. Mustalahti, C. Catassi, A. Reunanen et al., "The prevalence of celiac disease in Europe: results of a centralized, international mass screening project," Annals of Medicine, vol. 42, no. 8, pp. 587-595, 2010.

[3] M. J. Ehsani-Ardakani, M. RostamiNejad, V. Villanacci et al., "Gastrointestinal and non-gastrointestinal presentation in patients with celiac disease," Archives of Iranian Medicine, vol. 16, pp. 78-82, 2013.

[4] F. Zingone, M. Siniscalchi, P. Capone et al., "The quality of sleep in patients with coeliac disease," Alimentary Pharmacology and Therapeutics, vol. 32, no. 8, pp. 1031-1036, 2010.

[5] C. Ciacci, M. Cirillo, G. Auriemma, G. di Dato, F. Sabbatini, and G. Mazzacca, "Celiac disease and pregnancy outcome," American Journal of Gastroenterology, vol. 91, no. 4, pp. 718-722, 1996.
[6] P. H. R. Green and C. Cellier, "Medical progress: celiac disease," The New England Journal of Medicine, vol. 357, no. 17, pp. 17311743, 2007.

[7] G. R. Corazza, M. di Stefano, E. Mauriño et al., "Bones in coeliac disease: diagnosis and treatment," Best Practice \& Research Clinical Gastroenterology, vol. 19, pp. 453-465, 2005.

[8] J. F. Ludvigsson, F. Zingone, M. Fored, C. Ciacci, and M. Cirillo, "Moderately increased risk of urinary stone disease in patients with biopsy-verified coeliac disease," Alimentary Pharmacology and Therapeutics, vol. 35, no. 4, pp. 477-484, 2012.

[9] S. Tikkakoski, E. Savilahti, and K.-L. Kolho, "Undiagnosed coeliac disease and nutritional deficiencies in adults screened in primary health care," Scandinavian Journal of Gastroenterology, vol. 42, no. 1, pp. 60-65, 2007.

[10] G. Addolorato, E. Capristo, G. Ghittoni et al., "Anxiety but not depression decreases in coeliac patients after one-year gluten-free diet: a longitudinal study," Scandinavian Journal of Gastroenterology, vol. 36, no. 5, pp. 502-506, 2001.

[11] C. Hallert and J. Åström, "Psychic disturbances in adult coeliac disease. II. Psychological findings," Scandinavian Journal of Gastroenterology, vol. 17, pp. 21-24, 1982.

[12] D. Arigo, A. M. Anskis, and J. M. Smyth, "Psychiatric comorbidities in women with celiac disease," Chronic Illness, vol. 8, no. 1, pp. 45-55, 2012.

[13] M. A. Maxwell and D. A. Cole, "Weight change and appetite disturbance as symptoms of adolescent depression: toward an integrative biopsychosocial model," Clinical Psychology Review, vol. 29 , no. 3, pp. $260-273,2009$.

[14] A. E. Hamel, S. I. Zaitsoff, A. Taylor et al., "Body-related social comparison and disordered eating among adolescent females with an eating disorder, depressive disorder, and healthy controls," Nutrients, vol. 4, pp. 1260-1272, 2012.

[15] D. A. Leffler, M. Dennis, J. B. Edwards George et al., “The interaction between eating disorders and celiac disease: an exploration of 10 cases," International Journal of Eating Disorders, vol. 39, pp. 530-532, 2006.

[16] A. Karwautz and G. Wagner, "Coeliac disease and eating disorders-forgotten comorbidities?" Internal Medicine Journal, vol. 39, no. 11, pp. 784-785, 2009.

[17] G. R. Corazza, V. Villanacci, C. Zambelli et al., "Comparison of the interobserver reproducibility with different histologic criteria used in celiac disease," Clinical Gastroenterology and Hepatology, vol. 5, no. 7, pp. 838-843, 2007.

[18] P. Iovino, A. Pascariello, I. Russo et al., "Difficult diagnosis of celiac disease: diagnostic accuracy and utility of chromo-zoom endoscopy," Gastrointestinal Endoscopy, vol. 77, pp. 233-240, 2013.

[19] M. Setty, L. Hormaza, and S. Guandalini, "Celiac disease: risk assessment, diagnosis, and monitoring," Molecular Diagnosis and Therapy, vol. 12, no. 5, pp. 289-298, 2008.

[20] M. Bonaccio, A. di Castelnuovo, S. Costanzo et al., "Mass media information and adherence to Mediterranean diet: results from the Moli-sani study," International Journal of Public Health, vol. 57, pp. 589-597, 2012.

[21] J. Gormally, S. Black, S. Daston, and D. Rardin, "The assessment of binge eating severity among obese persons," Addictive Behaviors, vol. 7, no. 1, pp. 47-55, 1982.

[22] G. Finlayson, A. Arlotti, M. Dalton, N. King, and J. E. Blundell, "Implicit wanting and explicit liking are markers for trait binge eating. A susceptible phenotype for overeating," Appetite, vol. 57, no. 3, pp. 722-728, 2011. 
[23] A. E. Grupski, M. M. Hood, B. J. Hall et al., "Examining the binge eating scale in screening for binge eating disorder in bariatric surgery candidates," Obesity Surgery, vol. 23, pp. 1-6, 2013.

[24] D. M. Garner, M. P. Olmsted, Y. Bohr, and P. E. Garfinkel, “The eating attitudes test: psychometric features and clinical correlates," Psychological Medicine, vol. 12, no. 4, pp. 871-878, 1982.

[25] D. M. Garner, M. P. Olmstead, and J. Polivy, "Development and validation of a multidimensional eating disorder inventory for anorexia nervosa and bulimia," International Journal of Eating Disorders, vol. 2, no. 2, pp. 15-34, 1983.

[26] W. W. Zung, "A self-rating depression scale," Archives of General Psychiatry, vol. 12, pp. 63-70, 1965.

[27] J. White, K. White, and J. Razani, "Effects of endogenicity and severity on consistency of standard depression rating scales," Journal of Clinical Psychiatry, vol. 45, no. 6, pp. 260-261, 1984.

[28] C. D. Spielberger, R. L. Gorsuch, P. R. Lushene et al., Manual For the State-Trait Anxiety Inventory, Consulting Psychologists Press, Palo Alto, Calif, USA, 1983.

[29] L. R. Derogatis and K. L. Savitz, "The SCL-90-R and the Brief Symptom Inventory (BSI) in primary care," in Handbook of Psychological Assessment in Primary Care Settings, M. E. Maruish, Ed., vol. 236, pp. 297-334, Lawrence Erlbaum Associates, Mahwah, NJ, USA, 2000.

[30] W. Häuser, K.-H. Janke, B. Klump, M. Gregor, and A. Hinz, "Anxiety and depression in adult patients with celiac disease on a gluten-free diet," World Journal of Gastroenterology, vol. 16, no. 22, pp. 2780-2787, 2010.

[31] W. D. Chey, "The role of food in the functional gastrointestinal disorders: introduction to a manuscript series," The American Journal of Gastroenterology, vol. 108, pp. 694-697, 2013.

[32] A. Sainsbury, D. S. Sanders, and A. C. Ford, "Prevalence of irritable bowel syndrome-type symptoms in patients with celiac disease: a meta-analysis," Clinical Gastroenterology and Hepatology, vol. 11, pp. 359-365, 2013.

[33] N. Battista, A. di Sabatino, M. di Tommaso et al., "Altered expression of type-1 and type-2 cannabinoid receptors in celiac disease," PLoS One, vol. 8, no. 4, Article ID e62078, 2013. 


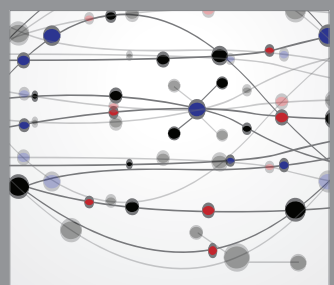

The Scientific World Journal
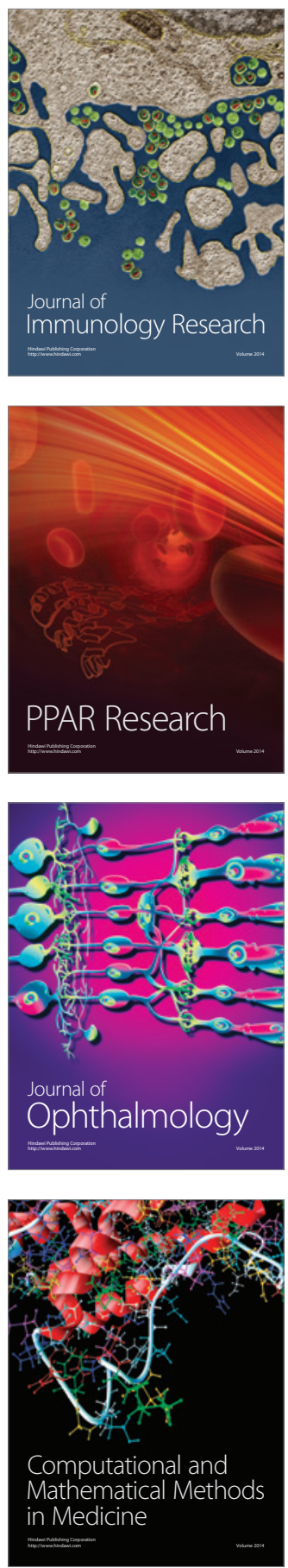

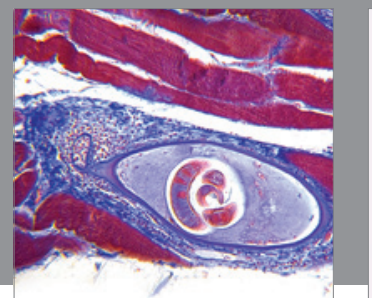

Gastroenterology

Research and Practice
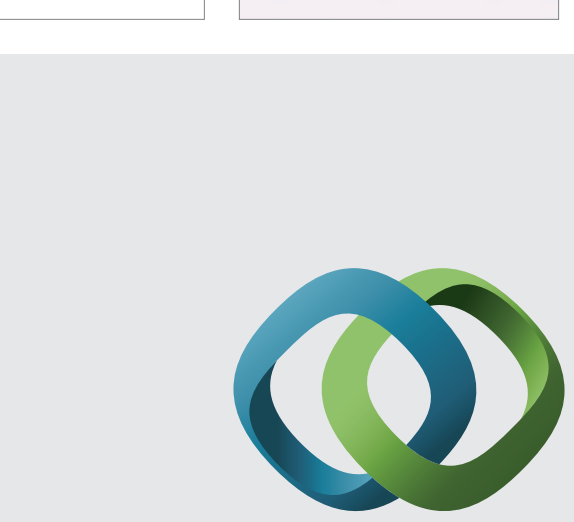

\section{Hindawi}

Submit your manuscripts at

http://www.hindawi.com
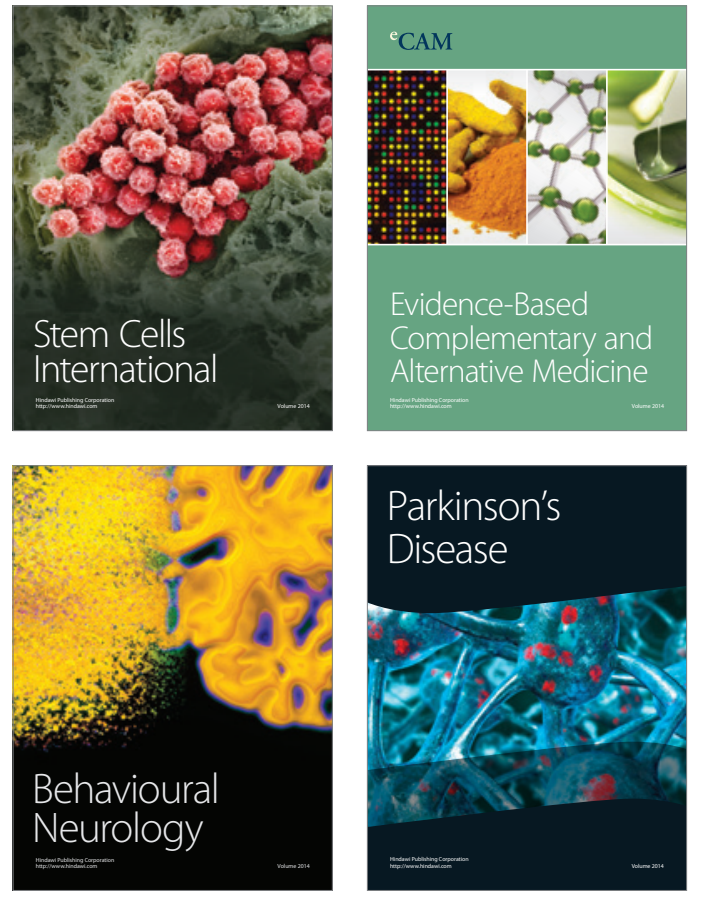
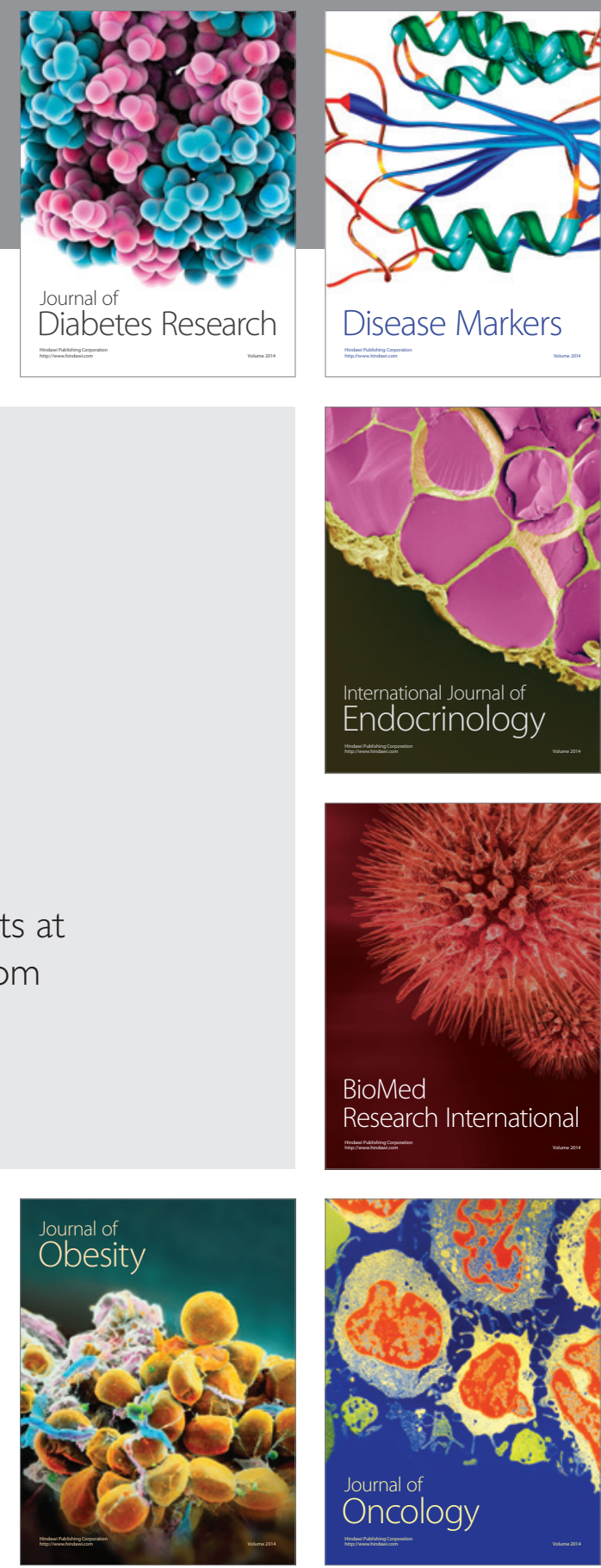

Disease Markers
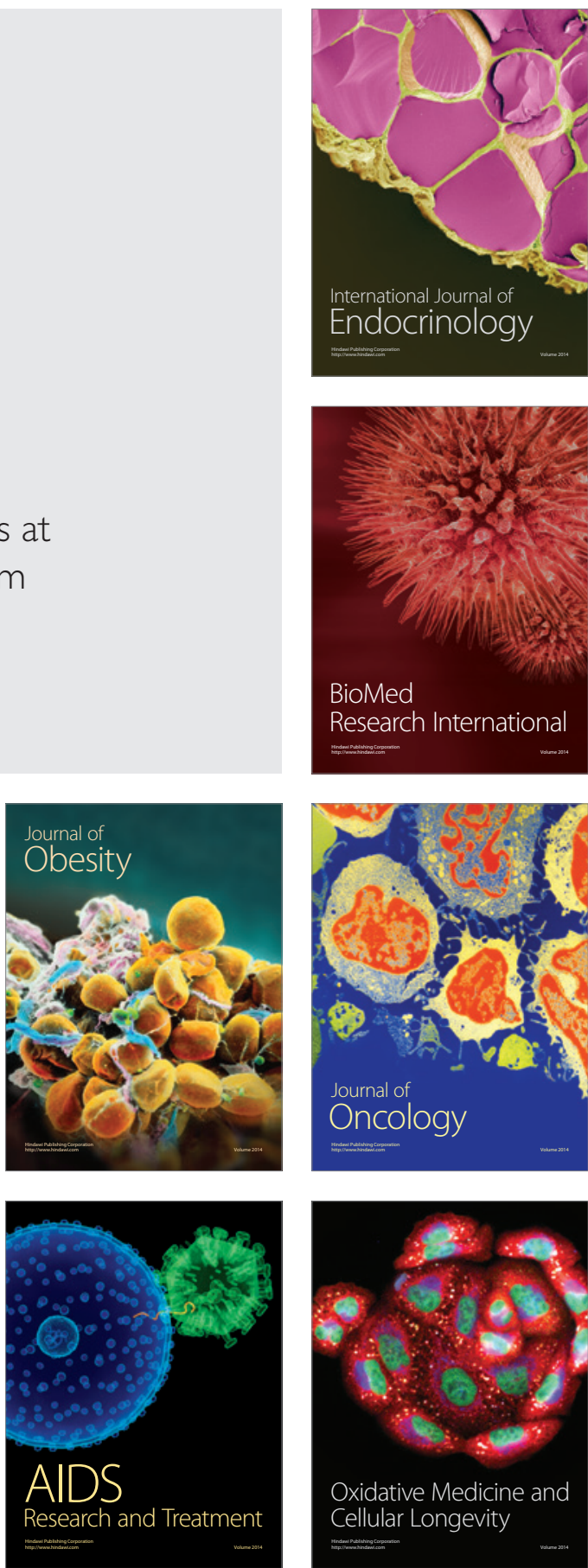